\title{
Application of Set Pair Analysis on QPE and Rain Gauge in Flood Forecasting
}

\author{
Zhiyuan Yin ${ }^{1, a}$, Fang Yang ${ }^{2, b}$, Tieyuan Shen ${ }^{1, c}$ \\ ${ }^{1}$ Hubei Key Laboratory for Heavy Rain Monitoring and Warning Research, Institute of Heavy Rain, \\ China Meteorological Administration, Wuhan, china, 430205 \\ 2Meteorological Information and Technology Support Center of Hubei Province, \\ Wuhan, china, 430074 \\ ayzy87888073@126.com, ${ }^{\mathrm{b}} \mathrm{cabbage} 0 @ 126 . c o m,{ }^{\mathrm{c}} \mathrm{ssttyy@126.com}$
}

\begin{abstract}
Key words: set pair analysis, quantitative precipitation estimation(QPE), weight calibration, flood forecasting

Abstract: This paper, taking Bailianhe reservoir basin on Hubei province as an example, firstly used the method of set pair analysis to calibrate the radar estimates rainfall combined with basin rain gauge. We obtained the optimal Z-I relationship of Bailianhe reservoir. Then, it presented a method of weight calibration based on radar estimation and rain gauge, combined with the advantage of the rainfall station at grid point observations and the radar to estimate the rainfall distribution. The method was used to analyze a rainfall process on Bailianhe basin in June 2004, compared with other rainfall estimation methods at last. The results showed that this method has certain effect on improving the precision of flood forecasting.
\end{abstract}

\section{Introduction}

Precipitation, as one of the important factors affecting the precision of flood forecasting, has always been the research hotspot. In recent years, with the remote sensing technology introduced into the hydrology forecasting, a lot of domestic and international hydrological and meteorological workers are committed to the research and application of radar rainfall estimation technique in hydrological forecasting ${ }^{[1]-[3]}$. For instance, Song Xingyuan ${ }^{[4]}$ used Calman filter and watershed measured rain gauge data combined with packet Z-I relationship to calibrate the radar estimate precipitation. It obtains the better result after the calibrated result input to the flood forecasting model. Zhang Liping $^{[5]}$ established the correlations expressions using basin rain gauge measured data and radar estimated precipitation. Then the radar estimates precipitation was input to the expression for each grid, obtaining the calibrated rainfall of the basin. It is helpful to improve the accuracy of rainfall estimation, compared with other rainfall estimation method. It is found that radar has better temporal and spatial resolution, and can obtain the instantaneous rainfall distribution on the surface of the river basin, which is better than that of precipitation obtained through interpolation. However, the rain gauge has better observation accuracy than that of radar at the single point. In order to make full use of radar and gauge in rainfall observation on their respective advantages, this paper presented a method based on weight calibration combined with radar and rain gauge data to estimate area precipitation distribution. To improve the precision of estimating areal rainfall, the method appropriately used the data of radar and rain gauge, with certain theoretical basis and simple calculation.

\section{Research Methods}

2.1 Determining the Coefficient of Z-I Relationship. This paper, by the most commonly-used method of the Z-I relationship, determined the radar rainfall field ${ }^{[6]-[7]}$. The expression is as follows: 


$$
\left\{\begin{array}{l}
Z=\int_{D_{\min }}^{D_{\max }} N(D) D^{6} d D \\
I=\frac{\pi}{6} \int_{D_{\min }}^{D_{\max }} N(D) \rho D^{3} V(D) d D
\end{array}\right.
$$

where $\mathrm{D}$ is the raindrops diameter; $\mathrm{D}_{\min }$ the minimum raindrops diameter; $\mathrm{D}_{\max }$ the minimum raindrops diameter; N(D) the distribution density of raindrops; V(D) the terminal falling velocity of raindrops; $\rho$ the raindrop density. Equation (1) shows $\mathrm{Z}$ and I are functions of $\mathrm{D}$, so there is a certain function relationship between $\mathrm{Z}$ and $\mathrm{I}$. It is assumed that $Z=A I^{b}$. Therefore, we can calculate the intensity of Precipitation I according to the radar reflectivity factor $\mathrm{Z}$, as long as the parameter $\mathrm{A}$ in the formula with the known value of $\mathrm{B}$. There are many methods to determine $\mathrm{A}$ and $\mathrm{b}$ values, including the actual statistics algorithm of Z-I relationship, the algorithm of probability matching between Z-I relationship and climate. It also directly applies the classical formula $Z=200 I^{\frac{14}{9}}$. This paper adopts a theory of uncertainty analysis method and the set pair analysis method to determine the coefficients $A$ and $\mathrm{B}^{[8]}$.

Zhao Keqin ${ }^{[9]}$ proposed a set pair analysis (SPA) method in 1989, and the theory of uncertainty can also describe the uncertainty from the same, the different and the opposite. The characteristics of set pair can be described by connection degree:

$$
\mu_{G-H}=\frac{S}{N}+\frac{F}{N} i+\frac{P}{N} j
$$

where $\mathrm{N}$ is the total number of samples; $\mathrm{S}$ the same number; $\mathrm{P}$ the opposite number; $\mathrm{F}$ the different number; I the difference uncertainty factor; $\mathrm{J}$ the opposite coefficient. During the calculation, the measured rainfall consists of a sequence $\mathrm{G}=\left[G_{0}, G_{1}, \cdots, G_{N}\right]$; radar rainfall estimates of a sequence $\mathrm{H}=\left[H_{0}, H_{1}, \cdots, H_{N}\right]$. So these two sequences can be composed of a sequence $\mathrm{G}-\mathrm{H}=$ $\left[G_{0}-H_{0}, G_{1}-H_{1}, \cdots, G_{N}-H_{N}\right]$. Then, we give the standard to distinguish the number of the same, the different and the opposition.

Making $C_{i}=\left|G_{i}-H_{i}\right|$, it is taken as the true value because of rainfall station are relatively accurate at the single point. When $C_{i} \leq 0.2 G_{i}, G_{i}-H_{i}$ is considered as the same; when $0.2 G_{i} \leq C_{i} \leq 0.25 G_{i}, G_{i}-H_{i}$ as the different; when $C_{i} \geq 0.25 G_{i}, G_{i}-H_{i}$ as the opposition. The objective function is established:

$$
\max \mu_{H-G}=\frac{S}{N}+\frac{F}{N} i+\frac{P}{N} j
$$

Making $i=0.5, j=-1$, we obtain the corresponding radar-estimated-rainfall sequences $\mathrm{H}$ after the program calculating and constantly adjusting the $\mathrm{A}$ and $\mathrm{b}$ values. Z-I relationship can be determined according to the set pair analysis to calculate the optimal value of the objective function.

2.2 Interpolation Method of Rain Gauge. The inverse distance square inverse method is used in rain gauge interpolation ${ }^{[10]}$. The main idea of the method is as follows:

The precipitation at arbitrary grid point without measured data can be determined by other reference rain gauge in the basin.

It is proportional to the unknown grid point rainfall and the other reference rain gauges.

The point to the reference station is inversely proportional to the distance. That is, 


$$
Z\left(x_{0}\right)=\frac{\sum_{i=1}^{n} Z\left(x_{i}\right) d\left(x_{0}, x_{i}\right)^{-2}}{\sum_{i=1}^{n} d\left(x_{0}, x_{i}\right)^{-2}}
$$

where $Z\left(x_{i}\right)$ is the reference station rainfall; $Z\left(x_{0}\right)$ the arbitrary grid rainfall; $d\left(x_{0}, x_{i}\right)$ the distance from unknown point to the known point.

2.3 Method based on Radar and Gauge Weight Calibration. Because of the rain gauge is accurate at the single point, we can assume that the approximate amount of each grid rainfall can be decided by the grid from the nearest one or several rain gauge within a certain range. On the other hand, the radar rainfall estimated at a single point has low accuracy than rain gauge, but the overall grasp of the situation is stronger than rain gauge in the whole basin. Therefore, under the condition of determined circumstance range of each grid rainfall, combined with the radar estimation rainfall, we further calibrate the grid rainfall. Then, it gives full play to the advantages of two kinds of rainfall estimation method, obtaining the ideal surface rainfall in basin. Based on the above ideas, this paper used the calibration formula as follows:

$$
P_{\text {calibrated }}=P_{\text {rain gauge }} \theta+P_{\text {radar }}(1-\theta)
$$

where $P_{\text {calibrated }}$ is the calibrated rainfall; $P_{\text {rain gauge }}$ the interpolation precipitation; $P_{\text {radar }}$ the radar estimate rainfall; $\theta$ the weight of each grid.

This paper adopted the similar method - inverse distance square inverse method - to calculate $\theta$. The basic idea is as follows: If there are $\mathrm{n}$ rain gauges in basin, the weight of given rain gauge to the unknown is

$$
\varepsilon\left(x_{i}, x_{0}\right)_{i}=\frac{d\left(x_{0}, x_{i}\right)^{-2}}{\sum_{i=1}^{n} d\left(x_{0}, x_{i}\right)^{-2}}
$$

The number with nearest distance to the given grid is $\mathrm{y}$, then

$$
\theta\left(x_{0}\right)=\sum_{i=1}^{y} \varepsilon\left(x_{i}, x_{0}\right)_{i} \quad y=1,2, \cdots, n
$$

To determine a reasonable value for the parameter $\theta$, this paper has performed the following treatment:

Making $y=i$, we can obtain the calibrated average rainfall, and $\bar{P}_{i}$ consists of a sequence $\bar{P}_{i}=\left[\bar{P}_{1}, \bar{P}_{2}, \cdots, \bar{P}_{n}\right] ;$ let $\bar{Q}_{i}=\bar{P}_{i+1}-\bar{P}_{i}, \bar{Q}_{i}=\left[\bar{Q}_{1}, \bar{Q}_{2}, \cdots, \bar{Q}_{n}\right] ;$ making $\bar{R}_{i}=\bar{Q}_{i}-\bar{Q}_{i+1}$, we can get $\bar{R}_{i}=\left[\bar{R}_{1}, \bar{R}_{2}, \cdots, \bar{R}_{n}\right]$. Through the analysis of the above three data sequences, it can be concluded: (1) With the increase of rain gauges, contribution to the basin of each grid rainfall also increases, so $\bar{P}_{i}$ is the increasing monotone function of y; (2) for the single grid, with the increase of rain gauges, the farther given rain gauge leads to the smaller contribution. Therefore, $\bar{Q}_{i}$ is the decreasing monotone function of $y$. It can be found that with the increase of $Y$, the change will be smaller and smaller. Considering this method uses rain gauge to control the range of rainfall and radar estimation rainfall to adjust the precision, we think the increasing number of rain gauges will no longer influence the grid rainfall when changes is very small. In order to determine an optimal y value, the paper gives a general standard: For the sequence $\bar{R}_{i}$, although without a strictly monotone decreasing function, the general trend is a gradually decreasing process. In general, the maximum value is $\bar{R}_{1}$, therefore the value of $\mathrm{y}$ is the optimal when $\bar{R}_{i}<0.05 \bar{R}_{1}$. 


\section{Example Analysis}

Taking Bailian River Basin in Hubei province as the research object, Bailianhe reservoir located in the middle of the Xishui River of the middle and lower of Yangtze River in the east of Hubei Province. There are 19 rain gauges in basin, whose area is $1,797 \mathrm{~km}^{2}$, long in the north and south, and narrow in the east and west. Using base data of the Wuhan Doppler Weather Radar (CINRAD/SA) $(30.5167 \mathrm{~N}, 114.3775$ degrees E, altitude $135.7 \mathrm{~m})$ in flood season of 2003 and the corresponding rain gauge data to optimize the coefficient of Z-I relationship, then we took a flood process simulation experiment in June 2004 at last.

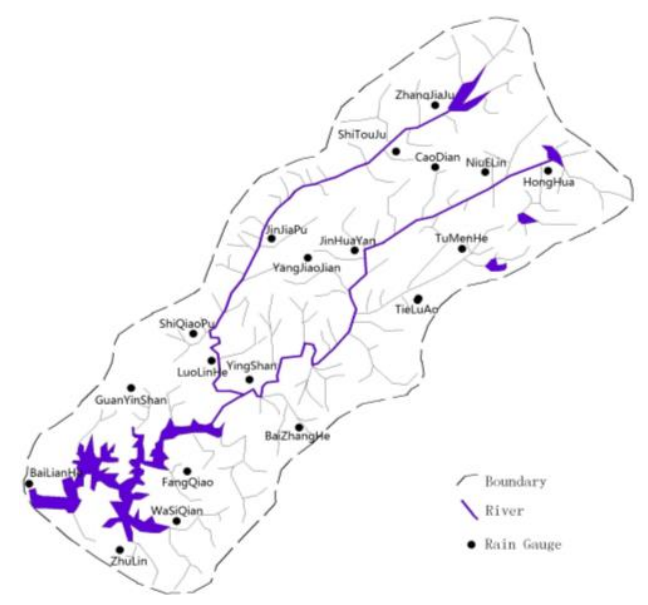

Fig.1. Picture of BaiLianHe basin

Table 1 the optimal parameters

\begin{tabular}{cccc}
\hline Parameter & $\mathrm{A}$ & $\mathrm{b}$ & $\mathrm{y}$ \\
\hline Value & 65 & 1.5 & 7
\end{tabular}

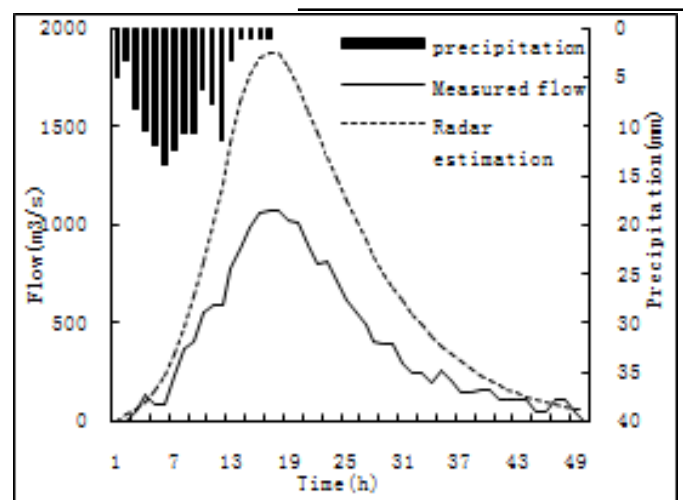

(a) Classical Z-I relationship estimating

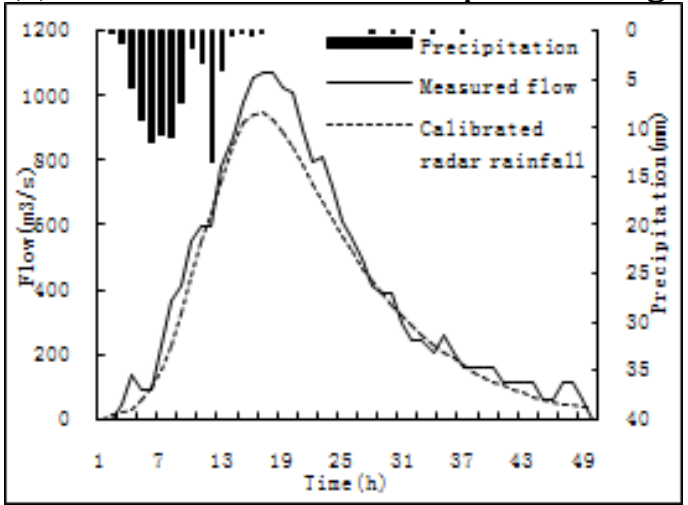

(c) Rain gauge interpolation

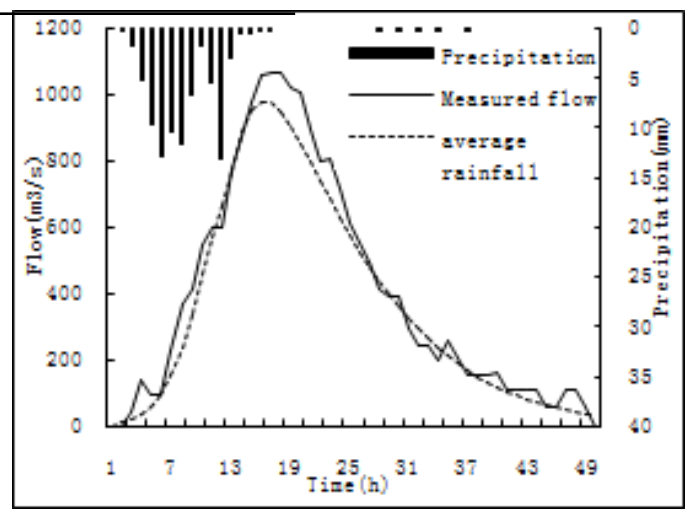

(b) Optimal Z-I relationship estimating

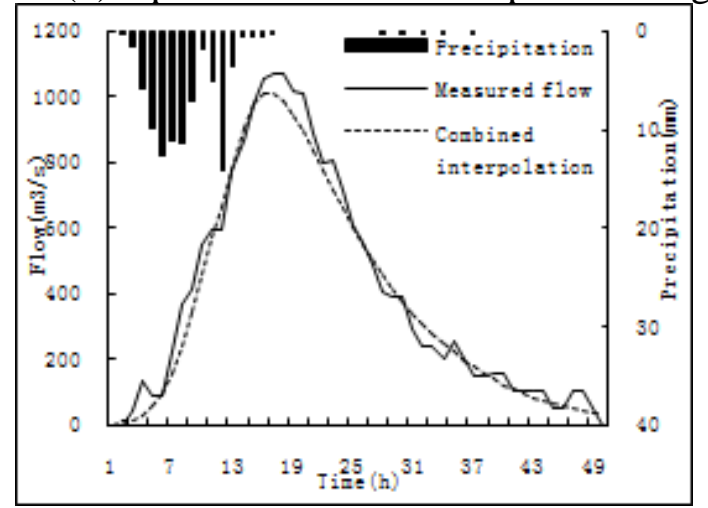

(d) Combined interpolation

Fig.2 Comparison of four kinds of estimating methods 
Table 2 Comparison of flood forecasting index with four kinds of rainfall estimation methods

\begin{tabular}{cccccc}
\hline method & $\begin{array}{c}\text { Measured } \\
\text { rain(mm) }\end{array}$ & $\begin{array}{c}\text { Average } \\
\text { rain(mm) }\end{array}$ & $\begin{array}{c}\text { Efficiency } \\
\text { coefficient(\%) }\end{array}$ & $\begin{array}{c}\text { Water balance } \\
\text { relative error } \\
(\%)\end{array}$ & $\begin{array}{c}\text { peak } \\
\text { time } \\
\text { error (h) }\end{array}$ \\
\hline $\begin{array}{c}\text { Classical Z-I } \\
\text { relationship }\end{array}$ & 88.1 & 119.5 & 42.69 & 75.38 & 0 \\
$\begin{array}{c}\text { Optimal Z-I } \\
\text { relationship }\end{array}$ & 88.1 & 83.5 & 90.83 & 8.34 & 1 \\
$\begin{array}{c}\text { Rain gauge } \\
\text { interpolation } \\
\text { Combined }\end{array}$ & 88.1 & 82.1 & 91.88 & 10.98 & 0 \\
interpolation & 88.1 & 85.5 & 93.79 & 4.90 & 0 \\
\hline
\end{tabular}

Figure 2 and Table 2 show the combined interpolation method has the best effect; rain gauge and optimal Z-I relationship have the same accuracy; the classic Z-I relationship estimate has the worst effect. The result of classic Z-I relationship estimation is too large, resulting in the relatively-larger flow forecasting. The result of optimized Z-I relationship estimation is improved to some degree, and the flood forecasting efficiency coefficient increases about $48 \%$, with the relative error of water balance reduced about 63\%. The calculated results are almost the same between the optimized Z-I relationship and rain gauge interpolation. The combined interpolation method has been improved based on the accuracy of optimized Z-I relationship, and the flood process efficiency coefficient increases about $3 \%$, with the water balance relative error reduced by about $3 \%$.

Table 3 each Group hourly cumulative relative and absolute error

\begin{tabular}{cccccc}
\hline Grouping & $\mathrm{NP}_{\mathrm{jk}}(\mathrm{mm})$ & $\mathrm{MP}_{\mathrm{jk}}(\%)$ & $\mathrm{NR}_{\mathrm{jk}}(\mathrm{mm})$ & $\mathrm{MR}_{\mathrm{jk}}(\%)$ & $\mathrm{LP}_{\mathrm{jk}}(\mathrm{mm})$ \\
\hline \multirow{5}{*}{ first group } & 5.38 & 59.82 & 8.40 & 93.38 & 9.0 \\
& 1.32 & 10.99 & 3.09 & 25.77 & 12.0 \\
& 3.52 & 17.61 & 5.01 & 25.07 & 20.0 \\
& 3.15 & 12.62 & 4.55 & 18.19 & 25.0 \\
& 8.94 & 55.90 & 1.78 & 11.11 & 16.0 \\
& 16.79 & 33.58 & 20.41 & 40.81 & 50.0 \\
& 7.16 & 34.11 & 4.90 & 23.35 & 21.0 \\
& 2.83 & 40.37 & 1.73 & 24.65 & 7.0 \\
& 8.27 & 31.81 & 11.15 & 42.87 & 26.0 \\
\hline \multirow{6}{*}{ second group } & 6.99 & 53.76 & 9.75 & 75.00 & 13.0 \\
& 13.28 & 47.42 & 7.23 & 25.83 & 28.0 \\
& 30.16 & 52.90 & 11.96 & 20.97 & 57.0 \\
& 24.46 & 35.97 & 11.48 & 16.88 & 68.0 \\
& 29.84 & 53.28 & 16.19 & 28.91 & 56.0 \\
& 15.59 & 28.88 & 15.05 & 27.88 & 54.0 \\
& 15.22 & 60.86 & 10.28 & 41.13 & 25.0 \\
& 8.13 & 67.74 & 2.29 & 19.06 & 12.0 \\
& 12.34 & 37.39 & 11.05 & 33.48 & 33.0 \\
& 52.06 & 66.74 & 27.81 & 35.65 & 78.0 \\
& 4.34 & 43.41 & 8.66 & 86.56 & 10.0 \\
\hline \multirow{6}{*}{ third group } & 10.21 & 46.39 & 14.59 & 66.34 & 22.0 \\
& 20.16 & 34.75 & 11.28 & 19.44 & 58.0 \\
& 14.75 & 16.39 & 15.90 & 17.67 & 90.0 \\
& 23.30 & 31.49 & 22.90 & 30.94 & 74.0 \\
& 28.64 & 34.51 & 15.64 & 18.84 & 83.0 \\
\hline
\end{tabular}


Table 3, cont.

\begin{tabular}{cccccc}
\hline & 20.26 & 33.76 & 17.95 & 29.92 & 60.0 \\
third group & 5.56 & 61.73 & 2.77 & 30.82 & 9.0 \\
& 27.03 & 67.58 & 20.51 & 51.28 & 40.0 \\
& 36.64 & 44.68 & 41.61 & 50.74 & 82.0 \\
& 7.18 & 39.92 & 12.26 & 68.11 & 18.0 \\
\hline & 24.54 & 53.34 & 8.60 & 18.70 & 46.0 \\
& 11.85 & 20.43 & 8.50 & 14.66 & 58.0 \\
fourth group & 11.36 & 16.00 & 12.59 & 17.73 & 71.0 \\
& 19.71 & 31.80 & 18.26 & 29.45 & 62.0 \\
& 13.28 & 28.87 & 12.17 & 26.45 & 46.0 \\
& 10.65 & 34.35 & 8.57 & 27.64 & 31.0 \\
& 3.00 & 37.51 & 2.93 & 36.61 & 8.0 \\
& 9.03 & 31.12 & 11.03 & 38.05 & 29.0 \\
summation(mm) & 28.54 & 38.06 & 17.20 & 22.93 & 75.0 \\
cumulative & 12.07 & 43.11 & 17.90 & 63.92 & 28.0 \\
\hline relative error $(\%)$ & 607.52 & & 485.92 & & 1610.0 \\
& 37.73 & & 30.18 & & 1 \\
\hline
\end{tabular}

Two methods with the same accuracy in Table 2 are further compared. Firstly, the number of the nineteen rainfall stations is followed by $1,2,3 \ldots \ldots, 18$ and 19. Then they are divided into four groups: the first group (1, 2, 3, 4 and 5); the second group (5, 6, 7, 8 and 9); the third group (10, 11, 12,13 and 14); the fourth group $(15,16,17,18$ and 19). There are five rain gauges in each group. There is a total of four groups of interpolation experiment, selecting a group does not participate in the interpolation of basin grid with fourteen site remains. Finally, the calculated values of five rain gauges in each group are compared with the measured result.

We make the measured data as $p_{i}$ ( $\mathrm{i}$ is site number as below). The result of grid rain gauge interpolation is $q_{i}$, and the result of grid radar rainfall estimation is $r_{i}$. In each group of the hourly cumulative relative error between rain gauge interpolation and measured data is $M P_{j k}=\sum_{i}\left|q_{i}-p_{i}\right| / \sum_{i} p_{i}$ ( $\mathrm{j}$ is time, and $\mathrm{k}$ is group number, as below), and cumulative absolute error is $N P_{j k}=\sum_{i}\left|q_{i}-p_{i}\right|$; in each group, the hourly cumulative relative error between radar estimation and measured data is $M R_{j k}=\sum_{i}\left|r_{i}-p_{i}\right| / \sum_{i} p_{i}$, and cumulative absolute error is $N R_{j k}=\sum_{i}\left|r_{i}-p_{i}\right|$; in each group, hourly-cumulative value is $L P_{j k}=\sum_{i} p_{i}$. Considering hourly rain below $1 \mathrm{~mm}$ is not included in the calculation process, and each group's hour is not consistent. The calculated results are shown in Table 3.

Table 3 shows rain gauge interpolation error fluctuated in $10.99-67.74 \%$. The time of relative error is bigger than $50 \%$, accounting for $27.5 \%$ of the total. The relative error is smaller than $40 \%$, accounting for $57.5 \%$ of the total. The relative error of combined interpolation fluctuate is in $11.11-93.38 \%$, and the time of relative error is bigger than $50 \%$, accounting for $20 \%$ of the total. The relative error is smaller than $40 \%$, accounting for $72.5 \%$ of the total. In addition, the time of the cumulative relative error of combined interpolation is smaller than rain gauge interpolation, accounting for $62.5 \%$ of the total. The combined interpolation is $30.18 \%$, better than the rain gauge interpolation's $37.73 \%$. It can be found despite the relative error changing range combined with interpolation is bigger, hourly-cumulative relative error in each group and cumulative relative error in all groups total times have smaller accumulated relative error than rain gauge interpolation. 


\section{Conclusions}

(1) It presented a kind of radar and rain gauge weight calibration method based on combination of advantages of the radar estimation in surface and rain gauge on the point observation. The method is very simple and convenient in the practical application.

(2) A comprehensive comparison of four kinds of rainfall estimation method was used in case analysis, with the optimal union interpolation algorithm. Rain gauge interpolation and Z-I relationship estimation have the same Precision, with the worst classical Z-I relationship estimation.

(3) According to the further comparing research, it is found that the relative error of the joint interpolation was $7 \%$ less than the rain gauge interpolation in the cumulative rainfall estimation.

(4) It only performs a case analysis, because of the few data and the shortly-selected time series, the conclusions need further verification.

\section{Acknowledgements}

This work has been supported by The Science and Technology Development Key Fund of Hubei Provincial Meteorological Bureau(2015Z02)

\section{References}

[1] LI, J. et al. Applications of "Frequency Matching" Method to Ensemble Precipitation Forecasts. Meteorological Monthly, 41(6):674-684, 2015.

[2] Yin, Z.Y. et al.Experiments of Bayesian probability flood forecasting based on the AREM model.Torrential Rain And Disasters 31(1):1-6,2012

[3] Zhao keqin. Set pair analysis and its prelimiary application. Hangzhou: Zhejiang science and Technology Press,2000:20-25

[4] Peters J C, Easidn D J.Runoff simulation using radar rainfall data.Water Resour, 32:753-760, 1997.

[5] Peng, T.et al. Hydrometeorology Forecast System of the Danjiangkou Basins in Hanjiang. JOURNAL OF APPLIED METEOROLOGICAL SCIENCE, 25(1): 112-119, 2014.

[6] Cui, C.G. et al. The Flood Forecast Test on QPF Coupling with Hydrological Model in Flood Season in Medium and Small Catchment. Meteorological Monthly 36(12):56-61, 2010.

[7] Wang, L.L. et al. Application of GRAPES Meteorological and Hydrological Coupled Model to Flood Forecast. Journal of Applied Meteorological Science 23(3):274-284,2012.

[8] Yin, Z.Y. et al. The preliminary experiment of genetic-neural network flood forecasting based on QPE and QPF.Torrential Rain And Disasters 32(4):360-368,2013.

[9] Zhao, J.B. et al. Precipitation Forecast Test of Township Based on Genetic Algorithm Neural Networks. Journal of Meteorological Research and Application 32(1):29-33,2011.

[10] Song, X.M. et al. Application of Xinanjiang Model Coupling with Artificial Neural Networks. Bulletin of Soil and Water Conservation 6(30):135-138,2010. 